\title{
Comment on "The Impact of Treatment with Continuous Positive Airway Pressure on Acute Carbon Monoxide Poisoning"
}

\author{
David Loughran; (i) Lewis Nelson
}

Rutgers New Jersey Medical School, Medical Toxicology, Newark, New Jersey USA

\author{
Correspondence: \\ David Loughran \\ Rutgers New Jersey Medical School - \\ Medical Toxicology \\ Newark, New Jersey 07103-2714 USA \\ E-mail: d.loughran@rutgers.edu
}

Conflicts of interest: The authors declare that there is no conflict of interest.

Keywords: carbon monoxide poisoning; continuous positive airway pressure ventilation; delayed neurological sequelae

Abbreviations:
CO: carbon monoxide
COHb: carboxyhemoglobin
CPAP: continuous positive airway pressure
ventilation
DNS: delayed neurological sequelae

Received: July 14, 2021

Accepted: July 24, 2021

doi:10.1017/S1049023X21001011

(C) The Author(s), 2021. Published by Cambridge University Press on behalf of the World Association for Disaster and Emergency Medicine.
Loughran D, Nelson L. Comment on "The impact of treatment with continuous positive airway pressure on acute carbon monoxide poisoning." Prehosp Disaster Med. 2021;36(6):805-806.

We read with interest the article by Caglar and colleagues ${ }^{1}$ and applaud their investigation into the novel use of continuous positive airway pressure ventilation (CPAP) for carbon monoxide $(\mathrm{CO})$ poisoning. We agree that adjunct treatments for $\mathrm{CO}$ poisoning are important and relevant areas of investigation given the general scarcity of hyperbaric chambers available for emergency use.

While we appreciated the authors' acknowledgement that this study was limited by a lack of evaluation of late neurological findings, these clinical outcomes are critical to assessing the efficacy of various treatments. Aside from the relatively immediate and consequential effects of organ ischemia and impaired cellular respiration associated with extremely high carboxyhemoglobin $(\mathrm{COHb})$ levels, the greatest risk from $\mathrm{CO}$ poisoning is from delayed neurological sequelae (DNS) ${ }^{2}$ Therefore, assessment of this outcome is a very important endpoint for determining effectiveness of treatment.

This study utilizes decreasing $\mathrm{COHb}$ levels to support the efficacy of CPAP as a treatment modality. While receiving supplemental normobaric oxygen clearly reduces $\mathrm{COHb}$ levels more rapidly than not receiving supplemental oxygen, ${ }^{3}$ more rapid reduction does not decrease the incidence of DNS. ${ }^{4}$ The cause of DNS remains incompletely understood but appears to be mediated by oxidative stress and activation of inflammatory cascades, lipid peroxidation, and immune-mediated injury in the central nervous system independent of ischemia. ${ }^{4,5}$ The development of DNS correlates poorly with $\mathrm{COHb}$ levels ${ }^{6}$ and the underlying inflammatory processes appear to continue long after the $\mathrm{COHb}$ level is below unacceptable levels.,7 Given our increasingly nuanced understanding of $\mathrm{CO}$ poisoning, the use of serial $\mathrm{COHb}$ levels in this and other studies as a surrogate marker is insufficient for determining the true clinical efficacy of proposed treatments such as CPAP.

We are also concerned about the study's use of symptoms including headache, nausea, dizziness, and weakness paired with $\mathrm{COHb}$ levels as surrogate markers of clinically important $\mathrm{CO}$ poisoning. These symptoms are subjective and fraught with potential confounders, and we are unaware of any studies confirming that the presence, absence, or resolution of these symptoms serve as useful indicators of $\mathrm{CO}$ toxicity or successful treatment. The presence or absence of initial symptoms has been found to correlate poorly with both initial $\mathrm{COHb}$ levels ${ }^{8}$ and the development of chronic sequelae. ${ }^{6}$

We urge the authors to continue their pursuit of this interesting and clinically relevant question in future studies, but with an approach more in-line with our current understanding of CO poisoning and pathophysiology. Inclusion of follow-up assessments for DNS and a reduction of focus on initial symptoms and $\mathrm{COHb}$ levels would be important changes to future investigations into determining the real efficacy and clinical impact of CPAP and other $\mathrm{CO}$ treatment modalities.

\footnotetext{
References

1. Caglar B, Serin S, Yilmaz G, Torun A, Parlak I. The impact of treatment with continuous positive airway pressure on acute carbon monoxide poisoning. Prehosp Disaster Med. 2019;34(6):588-591.

2. Weaver LK, Hopkins RO, Chan KJ, et al. Hyperbaric oxygen for acute carbon monoxide poisoning. $N$ Engl J Med. 2002;347(14):1057-1067.

3. Weaver LK, Howe S, Hopkins R, Chan KJ. Carboxyhemoglobin half-life in carbon monoxide-poisoned patients treated with 100\% oxygen at atmospheric pressure. Chest. 2000;117(3):801-808.

4. Weaver LK. Clinical practice. Carbon monoxide poisoning. N Engl J Med. 2009;360(12):1217-1225.
} 
5. Thom SR, Bhopale VM, Han ST, Clark JM, Hardy KR. Intravascular neutrophil activation due to carbon monoxide poisoning. Am J Respir Crit Care Med. 2006;174(11):1239-1248.

6. Weaver LK, Valentine KJ, Hopkins RO. Carbon monoxide poisoning: risk factors for cognitive sequelae and the role of hyperbaric oxygen. Am J Respir Crit Care Med. 2007;176(5):491-497.
7. Kuroda H, Fujihara K, Kushimoto S, Aoki M. Novel clinical grading of delayed neurologic sequelae after carbon monoxide poisoning and factors associated with outcome. Neurotoxicology. 2015;48:35-43.

8. Hampson NB, Hauff NM. Carboxyhemoglobin levels in carbon monoxide poisoning: do they correlate with the clinical picture? Am J Emerg Med. 2008;26(6): $665-669$. 